\title{
Nonfragile Gain-Scheduled Control for Discrete-Time Stochastic Systems with Randomly Occurring Sensor Saturations
}

\author{
Wangyan Li, ${ }^{1}$ Guoliang Wei, ${ }^{1}$ Hamid Reza Karimi, ${ }^{2}$ and Xiaohui Liu ${ }^{3}$ \\ ${ }^{1}$ Department of Control Science and Engineering, University of Shanghai for Science and Technology, Shanghai 200093, China \\ ${ }^{2}$ Department of Engineering, Faculty of Engineering and Science, University of Agder, N-4898 Grimstad, Norway \\ ${ }^{3}$ School of Computer Science and Technology, Nanjing University of Science and Technology, Nanjing 210094, China \\ Correspondence should be addressed to Guoliang Wei; guoliang.wei1973@gmail.com
}

Received 10 May 2013; Accepted 22 June 2013

Academic Editor: Jun $\mathrm{Hu}$

Copyright (C) 2013 Wangyan Li et al. This is an open access article distributed under the Creative Commons Attribution License, which permits unrestricted use, distribution, and reproduction in any medium, provided the original work is properly cited.

\begin{abstract}
This paper is devoted to tackling the control problem for a class of discrete-time stochastic systems with randomly occurring sensor saturations. The considered sensor saturation phenomenon is assumed to occur in a random way based on the time-varying Bernoulli distribution with measurable probability in real time. The aim of the paper is to design a nonfragile gain-scheduled controller with probability-dependent gains which can be achieved by solving a convex optimization problem via semidefinite programming method. Subsequently, a new kind of probability-dependent Lyapunov functional is proposed in order to derive the controller with less conservatism. Finally, an illustrative example will demonstrate the effectiveness of our designed procedures.
\end{abstract}

\section{Introduction}

In reality, virtually almost all dynamic systems are subject to stochastic perturbation, and stochastic model has been successfully established to describe many practical systems, such as economic systems, process control systems, networked control systems (NCSs), and sensor network. For several decades, the study of stabilization, control, and filtering problem has drawn many researchers' attention; some results can be found in [1-16]. On the other hand, time delays also serve as one of the main sources for poor performance and instability. Consequently, the stochastic control issue for time-delay systems has also been intensively investigated; see, for example, $[2,4,7,8,10,11,13,15]$.

The randomly occurring phenomenon is a newly emerged research topic which has drawn many researchers' attention; see, for example, $[1-3,5,6,8,9,12-16]$. It refers to these phenomena appearing in a random way based on a certain kind of probabilistic law including randomly occurring nonlinearities (RONs), missing measurements, randomly occurring actuator faults, randomly varying sensor delays (RVSDs), and randomly occurring sensor saturations (ROSSs), and so on. For more details about randomly occurring phenomena, the reader is referred to [9]. If not handled appropriately, these phenomena could cause a reduction of performance and/or launch a threat to the safety and reliability of the plant. Therefore, it is not surprising that various filtering and control techniques have been developed to deal with such randomly occurring phenomena, in addition to $H_{\infty}$ control [16]/filtering [12] and $H_{\infty}$ state estimation [1] methods. In [2], a robust sliding mode control has been designed for system with mixed time-delays, randomly occurring uncertainties, and RONs; while gain-constrained recursive filter approach has been used in [5] for system with probabilistic sensor delays, the extended Kalman filtering and quantized recursive filtering problem for system with missing measurements have been studied in $[3,6]$, respectively. Therefore, in this paper, the ROSS (one of the important randomly occurring phenomena) is studied by exploiting gain-scheduling method, which is another motivation of this paper.

Sensor saturation phenomenon is very common in practical engineering. It means that sensors cannot provide signals of unlimited amplitude due mainly to the physical or technological constraints. In another aspect, because of random occurrences of networked induced phenomena in networked control systems (NCSs), such as random sensor 
failures leading to intermittent saturation and sensor aging resulting in changeable saturation level, sensor saturation may occur in a random way. We consider this phenomenon as randomly occurring sensor saturation, which has received increasing attention, for instance, $[1,12]$. Reference [1] discussed the $H_{\infty}$ state estimation problem for discrete-time complex networks with ROSSs and RVSDs, while [12] turned to design an $H_{\infty}$ filter for system with ROSSs and missing measurements. However, to the best of authors' knowledge, rare published literature has dealt with ROSSs; therefore, this paper tries to flourish the research on this phenomenon by designing a nonfragile gain-scheduled controller.

Over the past decades, gain-scheduling method is one of the most popular methods of controller designing and has been extensively studied from theoretical and practical viewpoints; see, for example, $[8,14,15,17-19]$. The gainscheduling method is to design controller gains as functions of the scheduling parameters, which can update the controller with a set of tuning parameters in order to optimize the closed-loop performance when outside environment changes (e.g., the occurrences of a variety of randomly occurring phenomena). It should be noted that the designed gainscheduling controller has not only the constant part but also time-varying part which can be scheduled online according to the corresponding time-varying parameters; see $[8,14,15]$. Therefore, it will naturally lead to less conservatism than the conventional ones with fixed gains only.

On the other hand, it is well known that in order to get better performance of the system, an accuracy controller is needed to resist the impact by the uncertainties occurring in the course of the implementation of a designed controller. Such uncertainties can be due to the existence of parameter drift, round-off errors in numerical computation during controller implementation, and the safe-tuning margins provided for engineering application. In these cases, the nonfragile controller is a good choice, as it can tolerate some level of controller parameter variations; see [7, 20-22]. However, the controller with uncertainties and outside environment changes often occur simultaneously; unfortunately, few papers have tackled this phenomenon, and therefore, we proposed a nonfragile gain-scheduled controller in this paper to fill the gap by making a few first attempts to deal with this problem.

The main contributions of this paper are summarized as follows: (1) a new nonfragile gain-scheduled control problem is addressed for a class of discrete-time nonlinear stochastic systems with randomly occurring phenomenon; (2) a sequence of stochastic variables satisfying Bernoulli distribution is introduced to describe the time-varying features of the ROSSs; (3) a time-varying Lyapunov functional dependent on the saturation probability is proposed and applied to improve the performance of system; (4) the parameters of the nonfragile gain-scheduled controller can be adjusted online according to the saturating probability estimated through statistical tests.

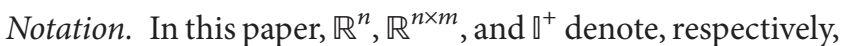
the $n$-dimensional Euclidean space, the set of all $n \times m$ real matrices, and the set of all positive integers. $|\cdot|$ refers to the Euclidean norm in $\mathbb{R}^{n}$. I denotes the identity matrix of compatible dimension. The notation $X \geq Y$ (resp., $X>$ $Y$ ), where $X$ and $Y$ are symmetric matrices, means that $X-Y$ is positive semidefinite (resp., positive definite). For a matrix $M, M^{T}$ and $M^{-1}$ represent its transpose and inverse, respectively. The shorthand $\operatorname{diag}\left\{M_{1}, M_{2}, \ldots, M_{n}\right\}$ denotes a block diagonal matrix with diagonal blocks being the matrices $M_{1}, M_{2}, \ldots, M_{n}$. In symmetric block matrices, the symbol $*$ is used as an ellipsis for terms induced by symmetry. Matrices, if they are not explicitly stated, are assumed to have compatible dimensions. In addition, $\mathbb{E}\{x\}$ and $\operatorname{Prob}\{y\}$ will, respectively, mean expectation of $x$ and probability of $y$.

\section{Problem Formulation}

Consider the following discrete-time nonlinear stochastic systems:

$$
\begin{array}{r}
x(k+1)=A x(k)+D x(k-d)+B u(k) \\
+N f(z(k))+E x(k) \omega(k), \\
x(k)=\rho(k), \quad k=-d,-d+1, \ldots, 0,
\end{array}
$$

where $x(k) \in \mathbb{R}^{n}$ is the state, $d$ is a constant delay and $z(k):=$ $G x(k)+G_{d} x(k-d), \omega(k)$ is a one-dimensional Gaussian white noise sequence satisfying $\mathbb{E}\{\omega(k)\}=0$ and $\mathbb{E}\left\{\omega^{2}(k)\right\}=\sigma^{2}$, and $\rho(k)$ is the initial state of the system. $A, B, D, E, N, G$, and $G_{d}$ are constant real matrices of appropriate dimensions and $B$ is of full column. The nonlinear function $f(\cdot)$ with $(f(0)=0)$ is assumed as nonlinear disturbance and satisfies the following sector-bounded condition:

$$
\left[f(z(k))-F_{1} z(k)\right]^{T}\left[f(z(k))-F_{2} z(k)\right] \leq 0,
$$

where $f(\cdot)$ belongs to the sector $\left[F_{1}, F_{2}\right], F_{1}$ and $F_{2}$ are given constant real matrices.

For the technique convenience, the nonlinear function $f(z(k))$ can be decomposed into a linear part and a nonlinear part as

$$
f(z(k))=f_{s}(z(k))+F_{1} z(k) ;
$$

then, from (3), we have

$$
f_{s}^{T}(z(k))\left(f_{s}(z(k))-F z(k)\right) \leq 0,
$$

where $F=F_{2}-F_{1}>0$.

The measurement output with sensor saturation is described as

$$
y(k)=\xi(k) \varrho(C x(k))+(1-\xi(k)) C x(k),
$$

where $C$ is a constant real matrix of appropriate dimensions and $\varrho(x)=\operatorname{sign}(x) \min \{1,|x|\}$. Here, the notation of "sign" means the signum function, and we use the notation $\varrho$ to denote saturation functions. Note that, without loss of generality, the saturation level is taken as unity.

According to the definition of the saturation function, we can get that the nonlinear function $\varrho$ satisfies $[\varrho(x)-$ $a x][\varrho(x)-x] \leq 0$ and $|x| \leq a^{-1}$, where $a$ is a positive scalar 
satisfying $0<a<1$, so the nonlinear function $\varrho(C x(k))$ satisfies $[\varrho(C x(k))-a C x(k)]^{T}[\varrho(C x(k))-C x(k)] \leq 0$, while $|a C x(k)| \leq 1$ and $a$ satisfies $0<a<1$.

The variable $\xi(k) \in \mathbb{R}$ is a random white sequence characterizing the probabilistic sensor saturation, which obeys the following time-varying Bernoulli distribution:

$$
\begin{gathered}
\operatorname{Prob}\{\xi(k)=1\}=\mathbb{E}\{\xi(k)\}=p(k), \\
\operatorname{Prob}\{\xi(k)=0\}=1-\mathbb{E}\{\xi(k)\}=1-p(k),
\end{gathered}
$$

where $p(k)$ is a time-varying positive scalar sequence and belongs to $\left[p_{1} p_{2}\right] \subseteq\left[\begin{array}{ll}0 & 1\end{array}\right]$ with $p_{1}$ and $p_{2}$ being the lower and upper bounds of $p(k)$, respectively. Throughout the paper, for simplicity, we assume that $\xi(k), \omega(k)$ and $\rho(k)$ are uncorrelated.

Remark 1. In many practical systems, especially in NCSs, the measurement output is often subject to ROSSs, and the Bernoulli distribution model has been proven to be a very flexible and effective way to model randomly occurring phenomenon; see, for example, [1-3, 5, 6, 8, 13-15]. Furthermore, in practical engineering, the occurring probability of sensor saturation phenomenon usually changes with time. Therefore, in this paper, the occurrence of sensor saturation is described by a random variable sequence $\xi(k)$ satisfying a time-varying instead of time-invariant Bernoulli distribution model, which will reduce the conservatism when used to deal with the systems with time-varying ROSSs.

In this paper, we are interested in designing the following nonfragile gain-scheduled static output feedback controller:

$$
u(k)=[K(p(k))+\Delta K] y(k),
$$

where $K(p(k))$ is the controller gain sequence to be designed and assumed as the following structure:

$$
K(p(k))=K_{0}+p(k) K_{u} ;
$$

for every time step $k, p(k)$ is the time-varying parameter of the controller gain, and $K_{0}$ and $K_{u}$ are the constant parameters of the controller gain to be designed, while $\Delta K$ is an unknown matrix of appropriate dimensions and represents the uncertainty in the controller, which is assumed to be of the form

$$
\Delta K=L H(k) M,
$$

where $L$ and $M$ are known constant matrices with the structured information of the uncertainty, and $H(k)$ is an unknown, real, and time-varying matrix with Lebesguemeasurable elements satisfying

$$
H^{T}(k) H(k) \leq I, \quad \forall k .
$$

Remark 2. Instead of using the information of system states, static output feedback control directly makes use of system outputs to design controllers, which has proven to be much simpler and easier to implement and has been extensively used in various kinds of engineering fields; for more details, we recommend some papers such as [23-27].
Remark 3. Owing to the pervasive existence of the uncertainties during controller implementation, an accuracy controller is needed to resist such an impact by the uncertainties, and the nonfragile controller has been proven to be an effective one; see, for example, [7, 20-22]. In another aspect, ROSSs are ubiquitous during the process of measurement, especially in NCSs, and gain-scheduling method has been successfully utilized to tackle with randomly occurring phenomenon in $[8,14,15]$. Therefore, in this paper, we design a nonfragile gain-scheduled static output feedback controller for nonlinear stochastic systems to deal with uncertainties and ROSSs simultaneously.

From the aforementioned, the closed-loop system with the nonfragile gain-scheduled controller is

$$
\begin{aligned}
x(k+1)= & A x(k)+D x(k-d)+B K(p(k)) \\
& \times[\xi(k) \varrho(C x(k))+(1-\xi(k)) C x(k)] \\
& +N f(z(k))+E x(k) \omega(k) .
\end{aligned}
$$

Before formulating the problem to be investigated, we first introduce the following stability concepts.

Definition 4. The closed-loop system (12) is said to be exponentially mean-square stable if, with $\omega(k)=0$, there exist constant $\alpha>0$ and $\tau \in(0,1)$ such that

$$
\mathbb{E}\left\{\left\|x_{k}\right\|^{2}\right\} \leq \alpha \tau^{k} \sup _{-d \leq i \leq 0} \mathbb{E}\left\{\left\|x_{i}\right\|^{2}\right\}, \quad k \in \mathbb{Q}^{+} .
$$

In this paper, our purpose is to design a probabilitydependent nonfragile gain-scheduled controller of the form (8) for the system (1) by exploiting a probability-dependent Lyapunov functional and LMI method such that, for all admissible sensor saturations and exogenous stochastic noise, the closed-loop system (12) is exponentially mean-square stable.

\section{Main Results}

The following lemmas will be used in the proofs of our main results in this paper.

Lemma 5 ((Schur complement) [28]). Given constant matrices $\Sigma_{1}, \Sigma_{2}, \Sigma_{3}$, where $\Sigma_{1}=\Sigma_{1}^{T}$ and $0<\Sigma_{2}=\Sigma_{2}^{T}$, then $\Sigma_{1}+\Sigma_{3}^{T} \Sigma_{2}^{-1} \Sigma_{3} \geq 0$ if and only if

$$
\left[\begin{array}{cc}
\Sigma_{1} & \Sigma_{3}^{T} \\
\Sigma_{3} & -\Sigma_{2}
\end{array}\right] \geq 0 \quad \text { or } \quad\left[\begin{array}{cc}
-\Sigma_{2} & \Sigma_{3} \\
\Sigma_{3}^{T} & \Sigma_{1}
\end{array}\right] \geq 0
$$

Lemma 6 (see [13]). Let the matrix $B \in R^{n \times m}$ be of fullcolumn rank. There always exist two orthogonal matrices $U \in$ $R^{n \times n}$ and $V \in R^{n \times n}$ such that $B=U\left[\begin{array}{c}\Sigma \\ 0\end{array}\right] V^{T}$ and $\Sigma=$ $\operatorname{diag}\left\{\sigma_{1}, \sigma_{2}, \ldots, \sigma_{m}\right\}$. If matrix $S$ has the following structure: $S=U\left[\begin{array}{cc}S_{11} & S_{12} \\ 0 & S_{22}\end{array}\right] U^{T}$, where $S_{11}, S_{12} \in R^{n \times(n-m)}$ and $S_{22} \in$ $R^{(n-m) \times(n-m)}$, then there exists a nonsingular matrix $R \in R^{m \times m}$ such that $S B=B R$. 
Lemma 7 ((S-procedure) [28]). For given matrices $Q=Q^{T}$, $H$, and $E$ with appropriate dimensions,

$$
Q+H F(k) E+E^{T} F^{T}(k) H^{T}<0
$$

holds for all $F(k)$ satisfying $F^{T}(k) F(k) \leq I$ if and only if there exists $\varepsilon>0$ such that

$$
Q+\varepsilon^{-1} H H^{T}+\varepsilon E^{T} E<0
$$

For convenience of presentation, we first consider the desired controller without uncertainty (i.e., $\Delta K=0$ ), and the result will be shown in Theorem 8 . Then, we design the nonfragile gain-scheduled controller in Theorem 10 based on the conclusion in Theorem 8 .

Theorem 8. Consider the discrete-time nonlinear stochastic systems with ROSSs (12). If there exist positive-definite matrices $Q(p(k))$ and $Q_{\tau}$, slack matrix $S$, and nonsingular matrices $Y(p(k))$ and $R$, such that the following LMIs hold:

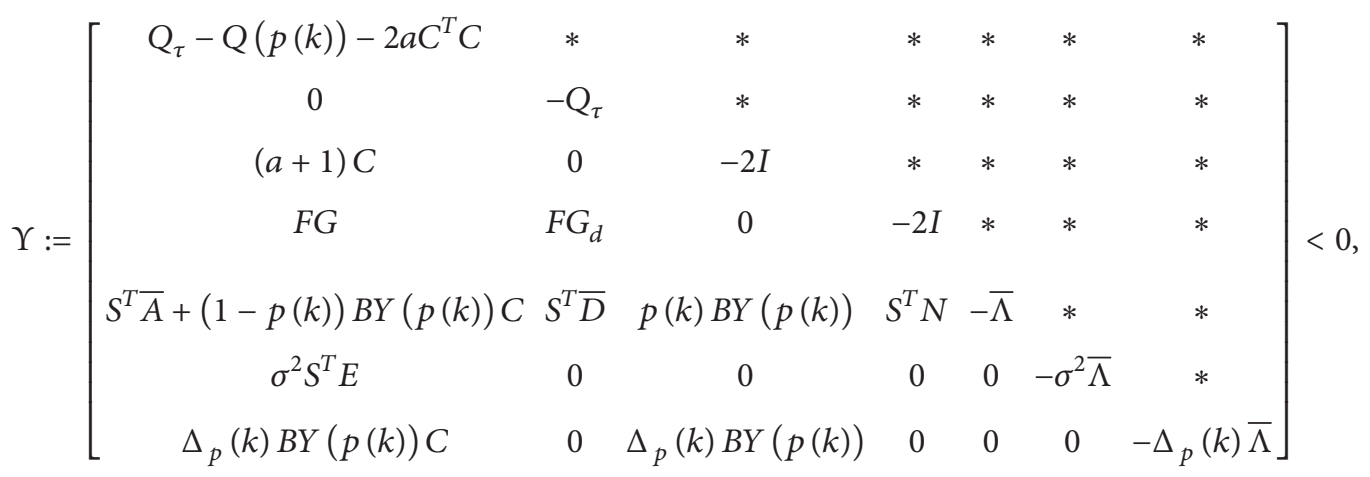

where

$$
\begin{gathered}
\bar{\Lambda}=-Q(p(k+1))+S+S^{T}, \quad \Delta_{p}(k)=p(k)(1-p(k)), \\
\bar{A}=A+N F_{1} G, \quad \bar{D}=D+N F_{1} G_{d}, \quad S^{T} B=B R, \\
R K(p(k))=Y(p(k)), \quad K(p(k))=R^{-1} Y(p(k)), \\
Y(p(k))=Y_{0}+p(k) Y_{u},
\end{gathered}
$$

in this case, the constant gains of the desired controller can be obtained as follows:

$$
K_{0}=R^{-1} Y_{0}, \quad K_{u}=R^{-1} Y_{u},
$$

and the closed-system (12) is then exponentially mean-square stable for all $p(k) \in\left[\begin{array}{ll}p_{1} & p_{2}\end{array}\right]$.

Proof. Define the Lyapunov functional

$$
V(k):=x^{T}(k) Q(p(k)) x(k)+\sum_{s=k-d}^{k-1} x^{T}(s) Q_{\tau} x(s)
$$

noting that $\mathbb{E}\{\xi(k)-p(k)\}=0, \mathbb{E}\{\omega(k)\}=0$, and $\mathbb{E}\left\{[\xi(k)-p(k)]^{2}\right\}=p(k)(1-p(k)) \triangleq \Delta_{p}(k)$, we can get

$$
\begin{aligned}
\mathbb{E}\{\Delta V(k)\}=\mathbb{E}\{ & x^{T}(k+1) Q(p(k+1)) x(k+1) \\
& -x^{T}(k)\left(Q(p(k))-Q_{\tau}\right) x(k) \\
- & \left.x^{T}(k-d) Q_{\tau} x(k-d)\right\}
\end{aligned}
$$

$$
\begin{aligned}
& =\mathbb{E}\{[A x(k)+p(k) B K(p(k)) \\
& \times[\varrho(C x(k))-C x(k)] \\
& +(\xi(k)-p(k)) B K(p(k)) \\
& \times[\varrho(C x(k))-C x(k)] \\
& +B K(p(k)) C x(k)+N f(z(k)) \\
& +D x(k-d)+E x(k) \omega(k)]^{T} Q(p(k+1)) \\
& \times[A x(k)+p(k) B K(p(k)) \\
& \times[\varrho(C x(k))-C x(k)] \\
& +(\xi(k)-p(k)) B K(p(k)) \\
& \times[\varrho(C x(k))-C x(k)] \\
& +B K(p(k)) C x(k)+D x(k-d) \\
& +N f(z(k))+E x(k) \omega(k)] \\
& -x^{T}(k) Q(p(k)) x(k)+x^{T}(k) Q_{\tau} x(k) \\
& \left.-x^{T}(k-d) Q_{\tau} x(k-d)\right\} \\
& \leq \mathbb{E}\{[(\bar{A}+(1-p(k)) B K(p(k)) C) x(k) \\
& +p(k) B K(p(k)) \varrho(C x(k))+\bar{D} x(k-d) \\
& \left.+N f_{s}(z(k))\right]^{T} \mathrm{Q}(p(k+1))
\end{aligned}
$$




$$
\begin{aligned}
\times & {[(\bar{A}+(1-p(k)) B K(p(k)) C) x(k)} \\
& +p(k) B K(p(k)) \varrho(C x(k))+\bar{D} x(k-d) \\
& \left.+N f_{s}(z(k))\right]+p(k)(1-p(k)) \\
\times & {[B K(p(k))(\varrho(C x(k))-C x(k))]^{T} } \\
& \times Q(p(k+1)) B K(p(k)) \\
& \times[\varrho(C x(k))-C x(k)] \\
+ & \sigma^{2} x^{T}(k) E^{T} Q(p(k+1)) E x(k) \\
- & x^{T}(k) Q(p(k)) x(k) \\
- & x^{T}(k-d) Q_{\tau} x(k-d) \\
+ & x^{T}(k) Q_{\tau} x(k)+2 f_{s}^{T}(z(k)) F G x(k) \\
+ & 2 f_{s}^{T}(z(k)) F G_{d} x(k-d) \\
- & 2 f_{s}^{T}(z(k)) f_{s}(z(k)) \\
- & 2 \varrho^{T}(C x(k)) \varrho(C x(k)) \\
+ & (2+2 a) \varrho^{T}(C x(k)) C x(k) \\
- & \left.2 a(C x(k))^{T} C x(k)\right\} .
\end{aligned}
$$

Denote the following matrix variables:

$$
\eta(k)=\left[\begin{array}{llll}
x^{T}(k) & x^{T}(k-d) & \varrho^{T}(C x(k)) & f_{s}^{T}(z(k))
\end{array}\right]^{T} ;
$$

then, it is obvious that

$$
\mathbb{E}\{\Delta V(k)\} \leq \mathbb{E}\left\{\eta^{T}(k) \Omega \eta(k)\right\},
$$

where

$$
\Omega=\left[\begin{array}{cccc}
\Omega_{1} & * & * & * \\
\Omega_{2} & \Omega_{3} & * & * \\
\Omega_{4} & \Omega_{5} & \Omega_{6} & * \\
\Omega_{7} & \Omega_{8} & \Omega_{9} & \Omega_{10}
\end{array}\right],
$$

$$
\left[\begin{array}{ccccccc}
Q_{\tau}-Q(p(k))-2 a C^{T} C & * & * & * & * & * & * \\
0 & -Q_{\tau} & * & * & * & * & * \\
(a+1) C & 0 & -2 I & * & * & * & * \\
F G & F G_{d} & 0 & -2 I & * & * & * \\
\bar{A}+(1-p(k)) B K(p(k)) C & \bar{D} & p(k) B K(p(k)) & N & -\Lambda & * & * \\
E & 0 & 0 & 0 & 0 & -\sigma^{-2} \Lambda & * \\
B K(p(k)) C & 0 & B K(p(k)) & 0 & 0 & 0 & -\Delta_{p}^{-1}(k) \Lambda
\end{array}\right]<0,
$$

If $\Omega \leq 0$, we can conclude the following matrix by Schur complement:

$$
\begin{aligned}
& \Omega_{1}=[\bar{A}+(1-p(k)) B K(p(k)) C]^{T} \\
& \times Q(p(k+1))[\bar{A}+(1-p(k)) B K(p(k)) C] \\
& +\sigma^{2} E^{T} Q(p(k+1)) E \\
& +\Delta_{p}(k)(B K(p(k)) C)^{T} Q(p(k+1)) \\
& \times B K(p(k)) C+Q_{\tau}-Q(p(k))-2 a C^{T} C, \\
& \Omega_{2}=\bar{D}^{T} Q(p(k+1))[\bar{A}+(1-p(k)) B K(p(k)) C] \text {, } \\
& \Omega_{3}=\bar{D}^{T} Q(p(k+1)) \bar{D}-Q_{\tau} \text {, } \\
& \Omega_{4}=p(k)(B K(p(k)))^{T} Q(p(k+1)) \\
& \times[\bar{A}+(1-p(k)) B K(p(k)) C] \\
& +\Delta_{p}(k)(B K(p(k)))^{T} Q(p(k+1)) \\
& \times B K(p(k)) C+(a+1) C, \\
& \Omega_{5}=p(k)(B K(p(k)))^{T} Q(p(k+1)) \bar{D}, \\
& \Omega_{6}=p^{2}(k)[B K(p(k))]^{T} Q(p(k+1)) \\
& \times B K(p(k))+\Delta_{p}(k)[B K(p(k))]^{T} \\
& \times Q(p(k+1)) B K(p(k))-2 I, \\
& \Omega_{7}=N^{T} Q(p(k+1)) \\
& \times[\bar{A}+(1-p(k)) B K(p(k)) C]+F G, \\
& \Omega_{8}=N^{T} Q(p(k+1)) \bar{D}+F G_{d}, \\
& \Omega_{9}=p(k) N^{T} Q(p(k+1)) B K(p(k)), \\
& \Omega_{10}=N^{T} Q(p(k+1)) N-2 I \text {. }
\end{aligned}
$$


where

$$
\Lambda=Q^{-1}(p(k+1)) ;
$$

by preforming the congruence transformation $\operatorname{diag}\{I, I, I, I$, $\left.S, \sigma^{2} S, \Delta_{p}(k) S\right\}$ to (25), we have

$$
\left[\begin{array}{ccccccc}
Q_{\tau}-Q(p(k))-2 a C^{T} C & * & * & * & * & * & * \\
0 & -Q_{\tau} & * & * & * & * & * \\
(a+1) C & 0 & -2 I & * & * & * & * \\
F G & F G_{d} & 0 & -2 I & * & * & * \\
S^{T} \bar{A}+(1-p(k)) S^{T} B K(p(k)) C & S^{T} \bar{D} & p(k) S^{T} B K(p(k)) & S^{T} N & -\widehat{\Lambda} & * & * \\
\sigma^{2} S^{T} E & 0 & 0 & 0 & 0 & -\sigma^{2} \widehat{\Lambda} & * \\
\Delta_{p}(k) S^{T} B K(p(k)) C & 0 & \Delta_{p}(k) S^{T} B K(p(k)) & 0 & 0 & 0 & -\Delta_{p}(k) \widehat{\Lambda}
\end{array}\right]<0,
$$

where

$$
\widehat{\Lambda}=S^{T} Q^{-1}(p(k+1)) S .
$$

From inequality

$$
S^{T} Q^{-1}(p(k+1)) S \geq S^{T}+S-Q(p(k+1)) \triangleq \bar{\Lambda},
$$

we can get

$$
\left[\begin{array}{ccccccc}
Q_{\tau}-Q(p(k))-2 a C^{T} C & * & * & * & * & * & * \\
0 & -Q_{\tau} & * & * & * & * & * \\
(a+1) C & 0 & -2 I & * & * & * & * \\
F G & F G_{d} & 0 & -2 I & * & * & * \\
S^{T} \bar{A}+(1-p(k)) S^{T} B K(p(k)) C & S^{T} \bar{D} & p(k) S^{T} B K(p(k)) & S^{T} N & -\bar{\Lambda} & * & * \\
\sigma^{2} S^{T} E & 0 & 0 & 0 & 0 & -\sigma^{2} \bar{\Lambda} & * \\
\Delta_{p}(k) S^{T} B K(p(k)) C & 0 & \Delta_{p}(k) S^{T} B K(p(k)) & 0 & 0 & 0 & -\Delta_{p}(k) \bar{\Lambda}
\end{array}\right]<0 .
$$

By using Lemma 6 , we have $S^{T} B=B R$, and denoting that $R K(p(k))=Y(p(k))$, then (30) can be written as (17); furthermore, we can know from Lemma 5 that $\Omega<0$ and, subsequently,

$$
\mathbb{E}\{\Delta V(k)\}<-\lambda_{\text {min }}(-\Omega) \mathbb{E}|\eta(k)|^{2},
$$

where $\lambda_{\min }(-\Omega)$ is the minimum eigenvalue of $(-\Omega)$. Finally, we can confirm from Lemma 1 in [13] that the closed-loop system is exponentially mean-square stable; then the proof of this theorem is complete.

Remark 9. The ROSSs have been studied in $[1,12]$ by constructing a concise and effective time-invariant Bernoulli distribution model; however, in many practical systems, ROSSs sometimes appear with time-varying probability. Therefore, in this case, we considered ROSSs satisfying time-varying Bernoulli distribution which is more reasonable in reality.
On the other hand, unlike other time-varying parameters discussed in gain-scheduling technique or parameter-dependent Lyapunov functional; see, for example, [17-19], the parameter $p(k)$ considered in this paper is the time-varying occurrence probability of ROSSs, based on which a new kind of controller is designed and a novel probability-dependent Lyapunov functional is proposed to reduce the potential conservatism.

Next, we are in a position to consider the nonfragile gainscheduled controller design for system (12) based on what we got in Theorem 8.

Theorem 10. Consider the discrete-time nonlinear stochastic systems with ROSSs (12) and the nonfragile gain-scheduled controller (8). If there exist positive-definite matrices $Q(p(k))$ and $Q_{\tau}$, slack matrix $S$, and nonsingular matrices $Y(p(k))$ 
and $R$, scalars $\varepsilon_{1}>0, \varepsilon_{2}>0$, LMIs (17), equations (18), and the following LMIs hold:

$$
\left[\begin{array}{ccccc}
\Upsilon & * & * & * & * \\
\varepsilon_{1} \Pi_{2}^{T} & -\varepsilon_{1} I & * & * & * \\
\Pi_{3} & 0 & -\varepsilon_{1} I & * & * \\
\varepsilon_{2} \Pi_{4}^{T} & 0 & 0 & -\varepsilon_{2} I & * \\
\Pi_{5} & 0 & 0 & 0 & -\varepsilon_{2} I
\end{array}\right]<0
$$

where

$$
\begin{aligned}
& \left.\Pi_{2}=\left[\begin{array}{llllll}
0 & 0 & 0 & 0 & {\left[(1-p(k)) S^{T} B L\right.}
\end{array}\right]^{T} \quad 0 \quad\left[\Delta_{p}(k) S^{T} B L\right]^{T}\right]^{T}, \\
& \Pi_{3}=\left[\begin{array}{lllllll}
M C & 0 & M & 0 & 0 & 0 & 0
\end{array}\right] \text {, } \\
& \left.\Pi_{4}=\left[\begin{array}{lllll}
0 & 0 & 0 & 0 & {\left[(2 p(k)-1) S^{T} B L\right.}
\end{array}\right]^{T} \quad 0 \quad 0\right]^{T}, \\
& \Pi_{5}=\left[\begin{array}{lllllll}
0 & 0 & M & 0 & 0 & 0 & 0
\end{array}\right] \text {; }
\end{aligned}
$$

in this case, the constant gains of the desired controller can be obtained as follows:

$$
K_{0}=R^{-1} Y_{0}, \quad K_{u}=R^{-1} Y_{u}
$$

and the closed-system (12) is then exponentially mean-square stable for all $p(k) \in\left[\begin{array}{ll}p_{1} & p_{2}\end{array}\right]$.

Proof. In order to get the nonfragile gain-scheduled controller, we replace the $K(p(k))$ with $K(p(k))+\Delta K$; then, $R K(p(k))=Y(p(k))$ can be written as $R[K(p(k))+\Delta K]=$
$Y(p(k))+\Delta Y, \Delta Y=R \Delta K=R L H(k) M$. Noting that $S^{T} B=$ $B R$, we can rewrite (17) as

$$
\begin{aligned}
\Upsilon & +\Pi_{2} H(k) \Pi_{3}+\Pi_{3}^{T} H^{T}(k) \Pi_{2}^{T} \\
& +\Pi_{4} H(k) \Pi_{5}+\Pi_{5}^{T} H^{T}(k) \Pi_{4}^{T}<0 .
\end{aligned}
$$

From Lemma 7, we know that a necessary and sufficient condition guaranteeing (35) is that there exist scalars $\varepsilon_{1}>0$, $\varepsilon_{2}>0$ such that

$$
\begin{aligned}
\Upsilon & +\varepsilon_{1} \Pi_{2} \Pi_{2}^{T}+\varepsilon_{1}^{-1} \Pi_{3}^{T} \Pi_{3} \\
& +\varepsilon_{2} \Pi_{4} \Pi_{4}^{T}+\varepsilon_{2}^{-1} \Pi_{5}^{T} \Pi_{5}<0 ;
\end{aligned}
$$

by using the knowledge of Schur complement, we can find that (36) is equivalent to (32). Now, the proof is complete.

Remark 11. In Theorem 10, a nonfragile gain-scheduled controller has been designed based on a set of LMIs. However, the LMIs are actually infinite owing to the time-varying parameter $p(k) \in\left[\begin{array}{ll}p_{1} & p_{2}\end{array}\right]$. In this case, the desired controller cannot be obtained directly due to the infinite number of LMIs. To handle such a problem, in the next theorem, we have to convert this problem to a computationally accessible one by assigning a specific form to $p(k)$. First of all, let us set $Q(p(k))=Q_{0}+p(k) Q_{u}$.

Theorem 12. Consider the discrete-time nonlinear stochastic system with ROSSs (12). If there exist positive-definite matrices $Q_{0}, Q_{u}$ and $Q_{\tau}$, slack matrix $S$ and nonsingular matrices $Y(p(k))$ and $R$, such that the following LMIs hold:

$$
\begin{aligned}
& \mathbb{M}^{i j l m}:=\left[\begin{array}{ccccc}
\Upsilon^{i j l m} & * & * & * & * \\
\varepsilon_{1} \Pi_{2}^{i j T} & -\varepsilon_{1} I & * & * & * \\
\Pi_{3} & 0 & -\varepsilon_{1} I & * & * \\
\varepsilon_{2} \Pi_{4}^{i T} & 0 & 0 & -\varepsilon_{2} I & * \\
\Pi_{5} & 0 & 0 & 0 & -\varepsilon_{2} I
\end{array}\right]<0 \\
& Y^{i j l m}:=\left[\begin{array}{ccccccc}
Q_{\tau}-Q^{i}(p(k))-2 a C^{T} C & * & * & * & * & * & * \\
0 & -Q_{\tau} & * & * & * & * & * \\
(a+1) C & 0 & -2 I & * & * & * & * \\
F G & F G_{d} & 0 & -2 I & * & * & * \\
S^{T} \bar{A}+\left(1-p_{i}\right) B Y^{m}(p(k)) C & S^{T} \bar{D} & p_{i} B Y^{m}(p(k)) & S^{T} N & -\bar{\Lambda}^{l} & * & * \\
\sigma^{2} S^{T} E & 0 & 0 & 0 & 0 & -\sigma^{2} \bar{\Lambda}^{l} & * \\
\Delta^{i j} B Y^{m}(p(k)) C & 0 & \Delta^{i j} B Y^{m}(p(k)) & 0 & 0 & 0 & -\Delta^{i j} \bar{\Lambda}^{l}
\end{array}\right] \text {, }
\end{aligned}
$$

where

$$
\begin{gathered}
\bar{\Lambda}=-Q_{0}-p_{l} Q_{u}+S+S^{T}, \quad \Delta^{i j}=p_{i}\left(1-p_{j}\right), \\
\bar{A}=A+N F_{1} G, \quad \bar{D}=D+N F_{1} G_{d}, \\
S^{T} B=B R, \quad R K(p(k))=Y(p(k)),
\end{gathered}
$$

$$
\begin{gathered}
K(p(k))=R^{-1} Y(p(k)) \\
Y^{m}(p(k))=Y_{0}+p_{m} Y_{u} \\
Q^{i}(p(k))=Q_{0}+p_{i} Q_{u}
\end{gathered}
$$$$
\left.\Pi_{2}^{i j}=\left[\begin{array}{lllll}
0 & 0 & 0 & 0 & {\left[\left(1-p_{i}\right) S^{T} B L\right.}
\end{array}\right]^{T} \quad 0\left[\Delta^{i j} S^{T} B L\right]^{T}\right]^{T},
$$ 


$$
\begin{aligned}
& \Pi_{3}=\left[\begin{array}{lllllll}
M C & 0 & M & 0 & 0 & 0 & 0
\end{array}\right], \\
& \left.\Pi_{4}^{i}=\left[\begin{array}{lllll}
0 & 0 & 0 & 0 & {\left[\left(2 p_{i}-1\right) S^{T} B L\right.}
\end{array}\right]^{T} \quad 0 \quad 0\right]^{T}, \\
& \Pi_{5}=\left[\begin{array}{lllllll}
0 & 0 & M & 0 & 0 & 0 & 0
\end{array}\right],
\end{aligned}
$$

in this case, the constant gains of the desired controller can be obtained as follows:

$$
K_{0}=R^{-1} Y_{0}, \quad K_{u}=R^{-1} Y_{u}
$$

and the closed-system (12) is then exponentially mean-square stable for all $p(k) \in\left[\begin{array}{ll}p_{1} & p_{2}\end{array}\right]$.

Proof. Firstly, set

$$
\alpha_{1}(k)=\frac{p_{2}-p(k)}{p_{2}-p_{1}}, \quad \alpha_{2}(k)=\frac{p(k)-p_{1}}{p_{2}-p_{1}}
$$

therefore, we have

$$
p(k)=\alpha_{1}(k) p_{1}+\alpha_{2}(k) p_{2}
$$

with $\alpha_{i}(k) \geq 0(i=1,2)$ and $\alpha_{1}(k)+\alpha_{2}(k)=1$. Similarly, let

$$
\beta_{1}(k)=\frac{p_{2}-p(k+1)}{p_{2}-p_{1}}, \quad \beta_{2}(k)=\frac{p(k+1)-p_{1}}{p_{2}-p_{1}},
$$

and we have

$$
p(k+1)=\beta_{1}(k) p_{1}+\beta_{2}(k) p_{2}
$$

with $\beta_{i}(k) \geq 0(i=1,2), \beta_{1}(k)+\beta_{2}(k)=1$. From the pervious transformation, we can easily get

$$
\begin{gathered}
Q(p(k))=\sum_{i=1}^{2} \alpha_{i}(k) Q^{i}(p(k)), \quad \bar{\Lambda}=\sum_{l=1}^{2} \beta_{l}(k) \bar{\Lambda}^{l} \\
Y(p(k))=\sum_{m=1}^{2} \alpha_{m}(k) Y^{m}(p(k))
\end{gathered}
$$

On the other hand, it is easy to find that

$$
\sum_{i, j, l, m=1}^{2} \alpha_{i}(k) \alpha_{j}(k) \alpha_{m}(k) \beta_{l}(k) \mathbb{M}^{i j l m}<0
$$

From (40)-(45), we can have that (32) in Theorem 10 is true; then the proof is now complete.

Remark 13. By using the methods proposed in the proof of Theorem 10, we choose 4 variables; then, it is easy to calculate the number of LMIs as $2^{4}$ depending on the upper and lower bound of $p(k)$.
TABLE 1: Computing results.

\begin{tabular}{cccc}
\hline$k$ & $p(k)$ & $Q(p(k))$ & $K(p(k))$ \\
\hline 0 & 0.5068 & {$\left[\begin{array}{cc}1.7244 & 0.7450 \\
0.7450 & 3.9564\end{array}\right]$} & {$\left[\begin{array}{cc}10.3093 & -12.4590 \\
-33.9040 & 40.8241\end{array}\right]$} \\
1 & 0.5082 & {$\left[\begin{array}{ll}1.7261 & 0.7443 \\
0.7443 & 3.9568\end{array}\right]$} & {$\left[\begin{array}{cc}10.3094 & -12.4590 \\
-33.9045 & 40.8242\end{array}\right]$} \\
2 & 0.4928 & {$\left[\begin{array}{cc}1.7070 & 0.7513 \\
0.7513 & 3.9522\end{array}\right]$} & {$\left[\begin{array}{cc}10.3078 & -12.4588 \\
-33.8992 & 40.8237\end{array}\right]$} \\
$\vdots$ & $\vdots$ & $\vdots$ & $\vdots$ \\
\hline
\end{tabular}

\section{An Illustrative Example}

In this section, the nonfragile gain-scheduled controller is designed for the discrete-time nonlinear stochastic systems with ROSSs.

The system parameters are given as follows:

$$
\begin{aligned}
& A=\left[\begin{array}{cc}
0.44 & 0 \\
0 & 0.81
\end{array}\right], \quad N=\left[\begin{array}{cc}
0.13 & 0.2 \\
0.28 & 0.33
\end{array}\right], \\
& B=\left[\begin{array}{cc}
0.01 & 0 \\
9.2 & 2.8
\end{array}\right], \quad C=\left[\begin{array}{cc}
0 & 0.19 \\
0.6 & 2.20
\end{array}\right] \text {, } \\
& D=\left[\begin{array}{ll}
0.02 & 0.14 \\
0.15 & 0.18
\end{array}\right], \quad F_{1}=\left[\begin{array}{cc}
0.06 & 0 \\
0 & 0.01
\end{array}\right] \text {, } \\
& F_{2}=\left[\begin{array}{cc}
0.1 & 0 \\
0 & 0.01
\end{array}\right], \quad G=\left[\begin{array}{ll}
0.08 & 0.12 \\
0.08 & 0.02
\end{array}\right] \text {, } \\
& G_{d}=\left[\begin{array}{ll}
0.01 & 0.09 \\
0.18 & 0.09
\end{array}\right], \quad E=\left[\begin{array}{ll}
0.3 & 0.19 \\
0.1 & 0.02
\end{array}\right] \text {, } \\
& L=\left[\begin{array}{cc}
0.01 & 0 \\
0 & 0.02
\end{array}\right], \quad M=\left[\begin{array}{cc}
0.1 & 0 \\
0 & 0.02
\end{array}\right] \text {, } \\
& H(k)=I, \quad p_{1}=0.49, \quad p_{2}=0.51, \\
& \sigma^{2}=1, \quad a=0.411, \quad \varepsilon_{1}=0.21, \quad \varepsilon_{2}=0.2 \text {. }
\end{aligned}
$$

Set the time-varying Bernoulli distribution sequences as $p(k)=p_{1}+\left(p_{2}-p_{1}\right)|\sin (k)|$, and the sector nonlinear function $f(u)$ is taken by

$$
f(u)=\frac{F_{1}+F_{2}}{2} u+\frac{F_{2}-F_{1}}{2} \sin (u),
$$

which satisfies (3). Also, select the initial state $\rho=\left[\begin{array}{ll}2 & -2\end{array}\right]^{T}$.

According to Theorem 12, the constant controller parameters $K_{0}, K_{u}$ can be obtained as follows:

$$
K_{0}=\left[\begin{array}{cc}
10.2565 & -12.4544 \\
-33.7306 & 40.8091
\end{array}\right], \quad K_{u}=\left[\begin{array}{cc}
0.1041 & -0.0090 \\
-0.3421 & 0.0296
\end{array}\right]
$$

Then, according to the measured time-varying probability parameters $p(k)$, the gain-scheduled controller gain $K(p(k))$ and parameter-dependent Lyapunov matrix $Q(p(k))$ can be calculated at every time step $k$ as in Table 1 . 


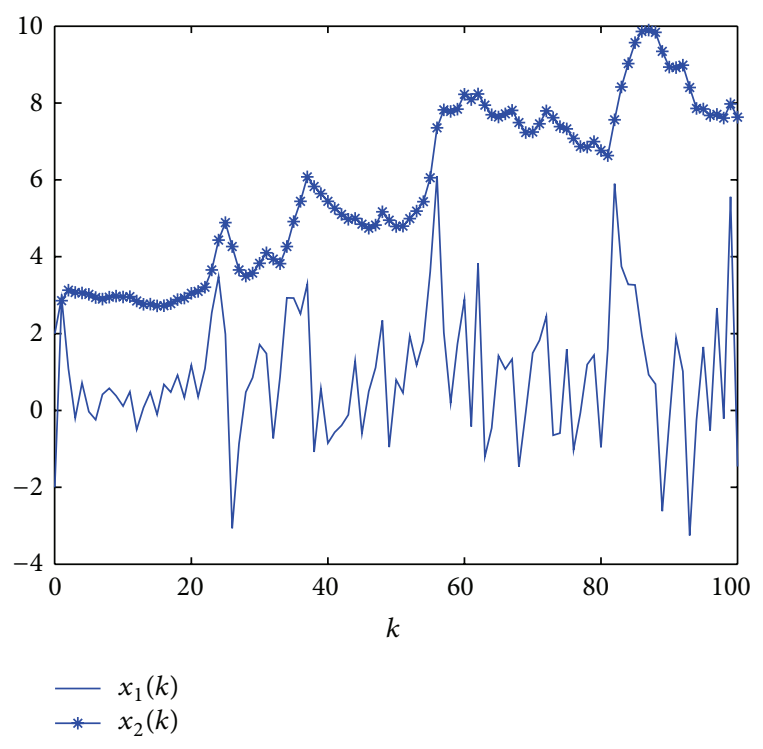

Figure 1: State evolution $x(k)$ of uncontrolled systems.

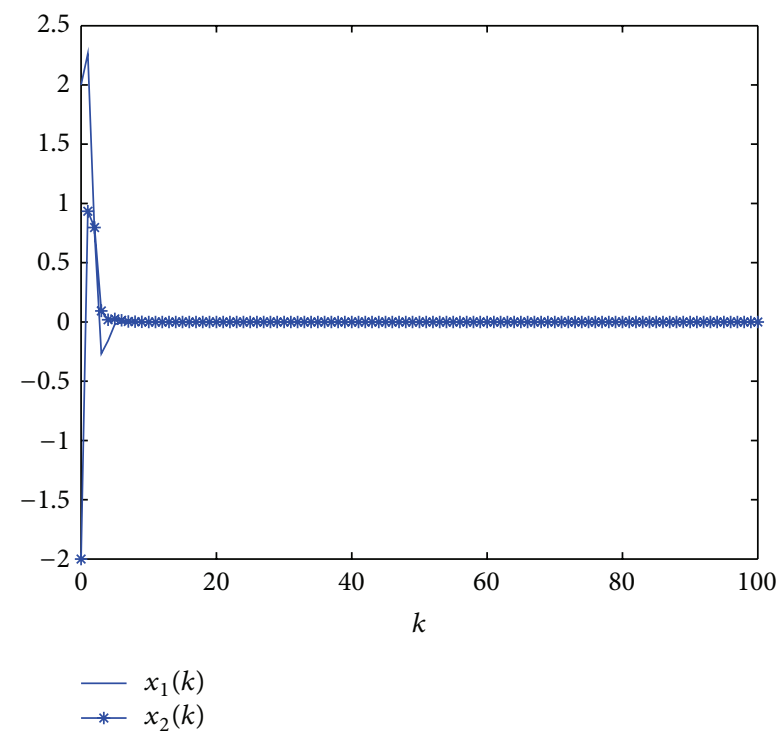

FIGURE 2: State evolution $x(k)$ of controlled systems.

Figure 1 gives the response curves of state $x(k)$ of uncontrolled systems. Figure 2 depicts the simulation results of state $x(k)$ of the controlled systems. The simulation results have illustrated our theoretical analysis.

\section{Conclusions}

In this paper, the nonfragile gain-scheduled control problem for a class of discrete stochastic systems with ROSSs is tackled, and the sensor saturation phenomenon is assumed to occur in a random way based on the time-varying Bernoulli distribution with measurable probability in real time. By employing probability-dependent Lyapunov functional, we design a nonfragile gain-scheduled controller with the gain including both constant and time-varying parameters such that, for all admissible sensor saturations, time-delays and noise disturbances, the closed-loop system is still exponentially mean-square stable. Furthermore, we can extend the main results to more complex and realistic systems, for instance, complex networks and systems with several kinds of randomly occurring phenomena simultaneously. Meanwhile, we can also consider corresponding control/filtering problems for time-varying systems with time-varying ROSSs, such as robust sliding mode control, quantized recursive filtering, or extended Kalman filtering. The related references can be found; see, for example, $[2,3,6]$.

\section{Acknowledgments}

This work was supported in part by the National Natural Science Foundation of China under Grant 61074016, the Program for Professor of Special Appointment (Eastern Scholar) at Shanghai Institutions of Higher Learning, the Program for New Century Excellent Talents in University under Grant NCET-11-1051, the Leverhulme Trust of the UK, the Alexander von Humboldt Foundation of Germany, and the Innovation Fund Project for Graduate Student of Shanghai under Grant JWCXSL1202.

\section{References}

[1] D. Ding, Z. Wang, B. Shen, and H. Shu, " $H_{\infty}$ state estimation for discrete-time complex networks with randomly occurring sensor saturations and randomly varying sensor delays," IEEE Transactions on Neural Networks and Learning Systems, vol. 23, no. 5, pp. 725-736, 2012.

[2] J. Hu, Z. Wang, H. Gao, and L. K. Stergioulas, "Robust sliding mode control for discrete stochastic systems with mixed time delays, randomly occurring uncertainties and randomly occurring nonlinearities," IEEE Transactions on Industrial Electronics, vol. 59, no. 7, pp. 3008-3015, 2012.

[3] J. Hu, Z. Wang, H. Gao, and L. K. Stergioulas, "Extended Kalman filtering with stochastic nonlinearities and multiple missing measurements," Automatica, vol. 48, no. 9, pp. 2007-2015, 2012.

[4] J. Hu, Z. Wang, Y. Niu, and L. K. Stergioulas, " $H_{\infty}$ sliding mode observer design for a class of nonlinear discrete time-delay systems: a delay-fractioning approach," International Journal of Robust and Nonlinear Control, vol. 22, no. 16, pp. 1806-1826, 2012.

[5] J. Hu, Z. Wang, B. Shen, and H. Gao, "Gain-constrained recursive filtering with stochastic nonlinearities and probabilistic sensor delays," IEEE Transactions on Signal Processing, vol. 61, no. 5, pp. 1230-1238, 2013.

[6] J. Hu, Z. Wang, B. Shen, and H. Gao, "Quantized recursive filtering for a class of nonlinear systems with multiplicative noises and missing measurements,' International Journal of Control, vol. 86, no. 4, pp. 650-663, 2013.

[7] L. Li and Y. Jia, "Non-fragile dynamic output feedback control for linear systems with time-varying delay," IET Control Theory \& Applications, vol. 3, no. 8, pp. 995-1005, 2009.

[8] W. Li, G. Wei, and L. Wang, "Probability-dependent static output feedback control for discrete-time nonlinear stochastic 
systems with missing measurements," Mathematical Problems in Engineering, vol. 2012, Article ID 696742, 15 pages, 2012.

[9] B. Shen, Z. Wang, J. Liang, and Y. Liu, "Recent advances on filtering and control for nonlinear stochastic complex systems with incomplete information: a survey," Mathematical Problems in Engineering, vol. 2012, Article ID 530759, 16 pages, 2012.

[10] H. Shu and G. Wei, " $H_{\infty}$ analysis of nonlinear stochastic timedelay systems," Chaos, Solitons \& Fractals, vol. 26, no. 2, pp. 637647, 2005.

[11] H. Shu, Z. Wang, and Z. Lü, "Global asymptotic stability of uncertain stochastic bi-directional associative memory networks with discrete and distributed delays," Mathematics and Computers in Simulation, vol. 80, no. 3, pp. 490-505, 2009.

[12] Z. Wang, B. Shen, and X. Liu, " $H_{\infty}$ filtering with randomly occurring sensor saturations and missing measurements," Automatica, vol. 48, no. 3, pp. 556-562, 2012.

[13] Z. Wang, F. Yang, D. W. C. Ho, and X. Liu, "Robust $H_{\infty}$ filtering for stochastic time-delay systems with missing measurements," IEEE Transactions on Signal Processing, vol. 54, no. 7, pp. 25792587, 2006.

[14] G. Wei, Z. Wang, and B. Shen, "Probability-dependent gainscheduled filtering for stochastic systems with missing measurements," IEEE Transactions on Circuits and Systems II, vol. 58, no. 11, pp. 753-757, 2011.

[15] G. Wei, Z. Wang, and B. Shen, "Probability-dependent gainscheduled control for discrete stochastic delayed systems with randomly occurring nonlinearities," International Journal of Robust and Nonlinear Control, vol. 23, no. 7, pp. 815-826, 2013.

[16] F. Yang, Z. Wang, Y. S. Hung, and M. Gani, " $H_{\infty}$ control for networked systems with random communication delays," IEEE Transactions on Automatic Control, vol. 51, no. 3, pp. 511-518, 2006.

[17] P. Apkarian and R. J. Adams, "Advanced gain-scheduling techniques for uncertain systems," IEEE Transactions on Control Systems Technology, vol. 6, no. 1, pp. 21-32, 1998.

[18] Y.-Y. Cao, Z. Lin, and Y. Shamash, "Set invariance analysis and gain-scheduling control for LPV systems subject to actuator saturation," Systems \& Control Letters, vol. 46, no. 2, pp. 137-151, 2002.

[19] W. J. Rugh and J. S. Shamma, "Research on gain scheduling," Automatica, vol. 36, no. 10, pp. 1401-1425, 2000.

[20] J. H. Park, "Robust non-fragile control for uncertain discretedelay large-scale systems with a class of controller gain variations," Applied Mathematics and Computation, vol. 149, no. 1, pp. 147-164, 2004.

[21] D. Peter, "Non-fragile controller design: an overview," in Proceedings of the American Control Conference, vol. 5, pp. 28292831, 1998.

[22] G.-H. Yang and W.-W. Che, "Non-fragile $H_{\infty}$ filter design for linear continuous-time systems," Automatica, vol. 44, no. 11, pp. 2849-2856, 2008.

[23] Y. Cao, J. Lam, and Y. Sun, "Static output feedback stabiliztion: an LMI approach," Automatica, vol. 34, no. 12, pp. 1641-1645, 1998.

[24] J. C. Geromel, C. C. de Souza, and R. E. Skelton, "Static output feedback controllers: stability and convexity," IEEE Transactions on Automatic Control, vol. 43, no. 1, pp. 120-125, 1998.

[25] I. N. Kar, "Design of static output feedback controller for uncertain systems," Automatica, vol. 35, no. 1, pp. 169-175, 1999.
[26] J. Qiu, G. Feng, and H. Gao, "Fuzzy-model-based piecewise $H_{\infty}$ static-output-feedback controller design for networked nonlinear systems," IEEE Transactions on Fuzzy Systems, vol. 18, no. 5, pp. 919-934, 2010.

[27] V. L. Syrmos, C. T. Abdallah, P. Dorato, and K. Grigoriadis, "Static output feedback-a survey," Automatica, vol. 33, no. 2, pp. 125-137, 1997.

[28] S. Boyd, L. El Ghaoui, E. Feron, and V. Balakrishnan, Linear Matrix Inequalities in Systems and Control Theory, Society for Industrial and Applied Mathematics (SIAM), Philadelphia, Pa, USA, 1994. 


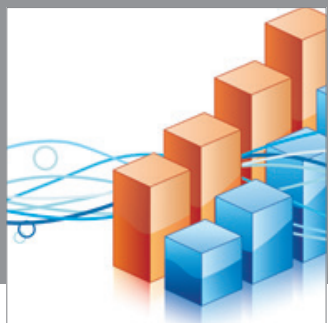

Advances in

Operations Research

mansans

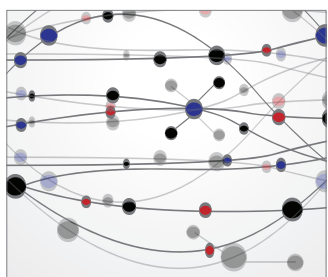

The Scientific World Journal
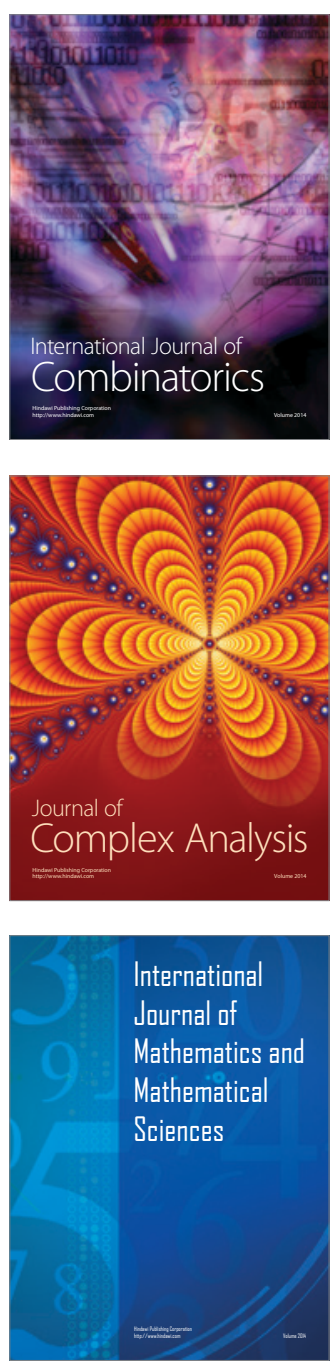
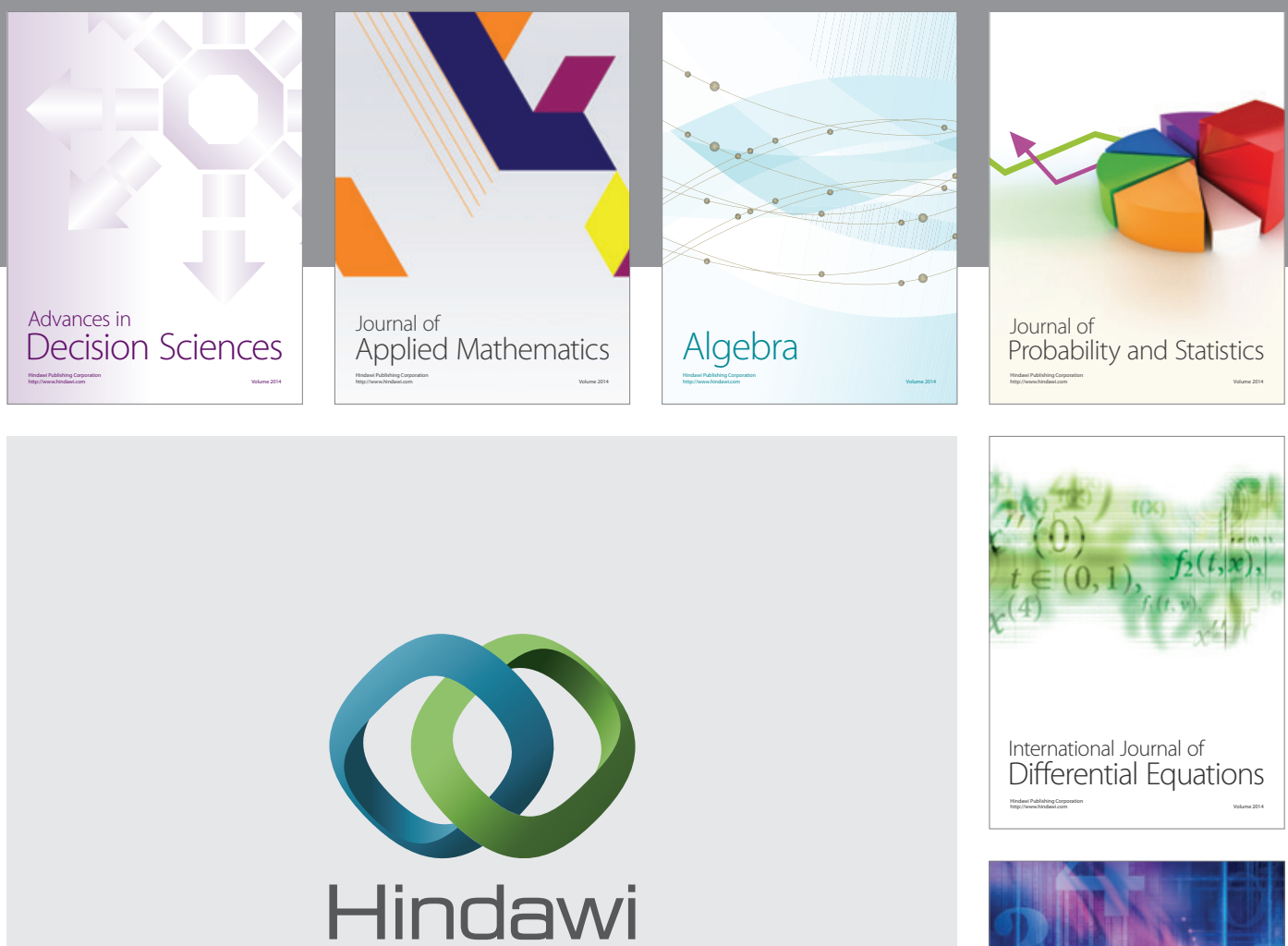

Submit your manuscripts at http://www.hindawi.com
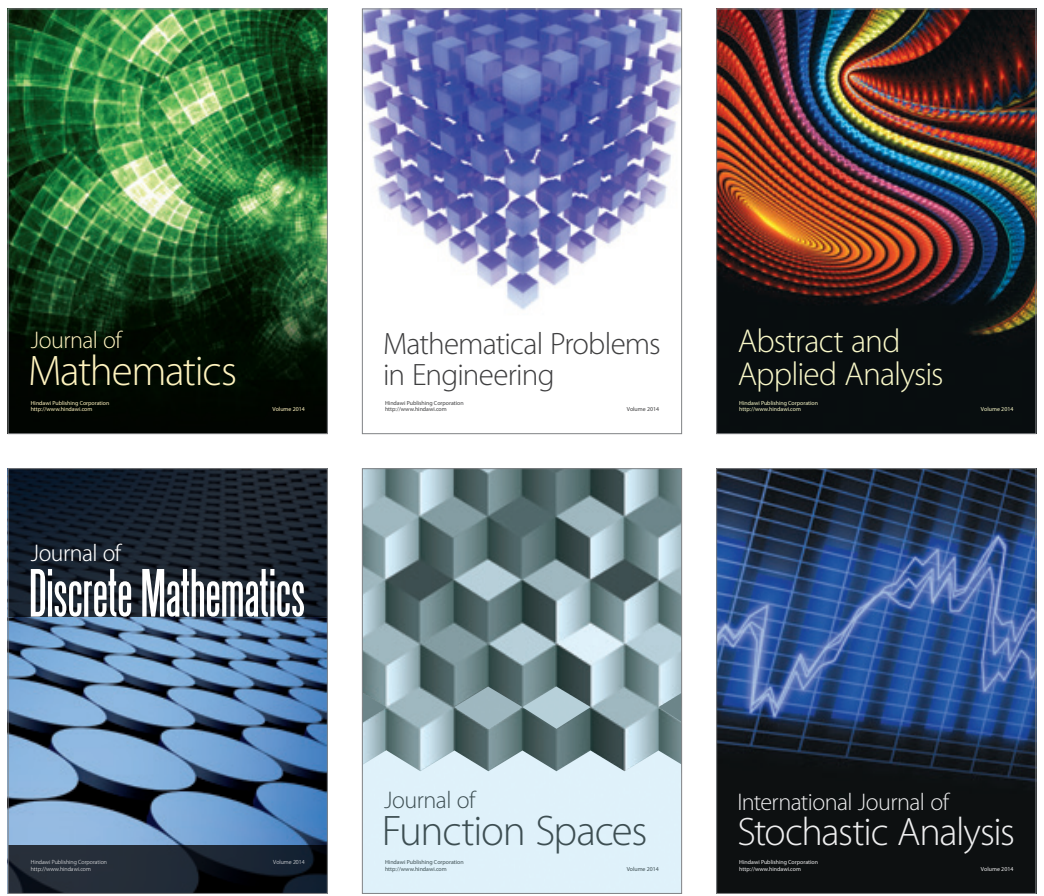

Journal of

Function Spaces

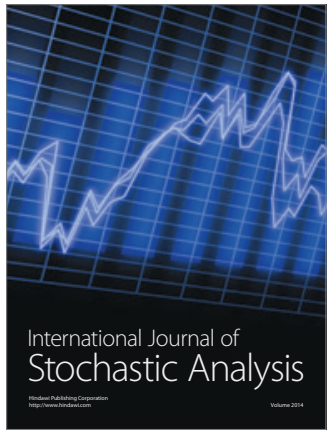

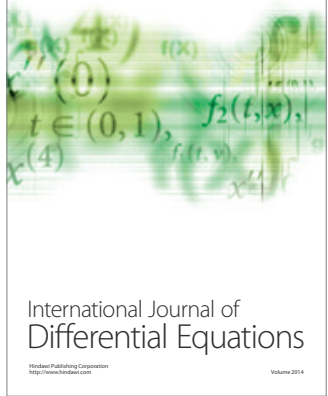
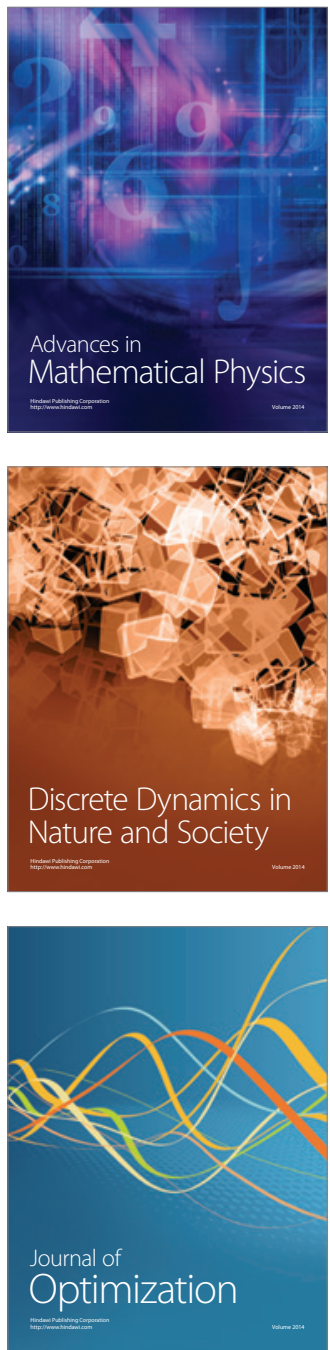